\title{
Brownian noise in radiation-pressure-driven micromechanical oscillators
}

\author{
Hossein Rokhsari and Mani Hossein-Zadeh \\ Department of Applied Physics, California Institute of Technology, Pasadena, California 91125 \\ Ali Hajimiri \\ Department of Electrical Engineering, California Institute of Technology, Pasadena, California 91125
}

Kerry Vahala ${ }^{a}$

Department of Applied Physics, California Institute of Technology, Pasadena, California 91125

(Received 1 May 2006; accepted 21 November 2006; published online 27 December 2006)

\begin{abstract}
The authors demonstrate Brownian-noise-limited operation of an optomechanical oscillator, wherein mechanical oscillations of a silica optical microcavity are sustained by means of radiation pressure. Using phase noise measurement above threshold, it has been shown that the short-term linewidth of mechanical oscillations is fundamentally broadened, limited by thermal equipartition of energy.

(C) 2006 American Institute of Physics. [DOI: 10.1063/1.2424276]
\end{abstract}

A class of mechanical oscillators has recently been demonstrated, functioning in the optomechanical domain and acquiring gain directly from optical fields. ${ }^{1-3}$ For a radial mechanical eigenmode of the microtoroidal cavity forced by the radiation pressure of the circulating optical power, the equation of motion can be written as 3,4

$$
\ddot{r}(t)+\gamma_{0} \dot{r}(t)+\Omega^{2} r(t)=F_{r p}=\frac{2 \pi n}{c m_{\mathrm{eff}}} P_{\text {circ }}(r) .
$$

The "mechanical gain" (or damping in the case of cooling, see Ref. 2) emerges from the term on the right hand side of the equation and offsets mechanical loss in the damped, harmonic equation of motion. For example, for below threshold operation, the intrinsic loss rate in Eq. (1) is replaced by an effective loss rate given by ${ }^{1}$

$$
\begin{aligned}
& \gamma_{\text {eff }}=\gamma_{0}-g, \\
& g=\gamma_{0} \frac{P_{j}}{P_{\text {th }}},
\end{aligned}
$$

where $P_{j}$ is the optical input power and $P_{\text {th }}$ is the threshold power for regenerative oscillations and can be calculated using the optical and mechanical properties of the system. ${ }^{2}$ Note that $g$ is the small-signal optomechanical gain at the operating point so $\Delta g \ll g$ during the oscillation.

This heretofore unknown category of hybrid oscillators is based on cooperative interaction of optical and mechanical resonators colocated within the same device. The oscillators consist of a microscale silica structure in the shape of a toroid that allows both resonant circulation of long-lived photons (coupled to the structure from an optical waveguide) as well as mechanical vibrations at characteristic radio frequencies. The coupling of these distinct mechanical and optical motions is provided by radiation pressure of the circulating photons. Due to the high- $Q$ nature of the toroidal optical modes, this coupling is sufficient to create regenerative rf mechanical oscillations with only microwatts of input optical power. Note that here the circulating optical power provides gain for the mechanical oscillations as well as the ability to probe the radius of the toroidal cavity through optical inter-

\footnotetext{
a) Author to whom correspondence should be addressed; electronic mail: vahala@caltech.edu; URL: www.vahala.caltech.edu
}

ference. The radial mechanical vibration modulates the optical resonant frequency and manifests itself as the amplitude modulation of the transmitted optical power. In this letter we investigate the fundamental linewidth of this oscillator by measuring the associated phase noise spectral density. The noise limit involved here is important to applications of these devices as optical clocks and in quantum optics. ${ }^{5}$ We are able to observe the thermomechanical noise, also referred to as Brownian noise, and the experimental results are in excellent agreement with the general, inverse-power linewidth narrowing principle in oscillators. ${ }^{6-8}$

Prior theoretical studies of regenerative oscillator performance, when embedded in a thermal bath at temperature $T$, predict that the fundamental oscillation linewidth (that is limited by thermal equipartition of energy) becomes increasingly narrowed as the oscillation power is boosted in the system. The general, inverse-power narrowing of oscillation linewidth is given by the following expression: ${ }^{8}$

$$
\delta \nu=\frac{\pi k_{B} T}{P}(\Delta \nu)^{2},
$$

where $\delta \nu$ is the oscillation linewidth, $\Delta \nu$ is the intrinsic linewidth of the resonator (in the present case the natural linewidth of the mechanical eigenmode), $P$ is the output power of the oscillator, and $k_{B}$ is the Boltzmann constant. Derivation of this formula has been given in Ref. 8 in the context of electrical $(R L C)$ oscillators. In any regenerative oscillator, the linewidth results from the cumulative fluctuations in phase caused by the noise power added to the oscillating field. In the present case, this noise is thermal in nature and its net impact on phase and linewidth is smaller for larger oscillation amplitudes. This intuitively helps to explain why the linewidth varies inversely with oscillation energy (or power) and linearly with the thermal energy $k_{B} T$.

In a mechanical resonator the dissipated (output) power $P$ can be related to the stored mechanical energy $(P$ $=2 \pi \nu E / Q=2 \pi \Delta \nu E$, where $E$ is the stored energy). So Eq. (3) may be restated as

$$
E=\frac{1}{2} k_{B} T \frac{\Delta \nu}{\delta \nu} .
$$

Equation (4) implies that the linewidth narrowing factor $(\Delta \nu / \delta \nu)$ scales proportionally to the oscillation energy. 


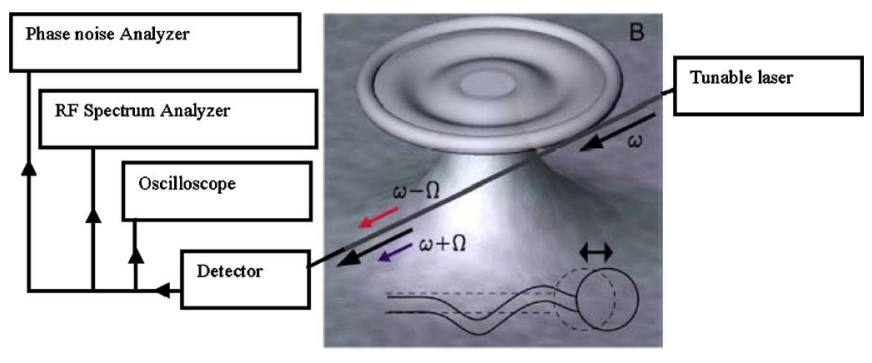

FIG. 1. (Color online) Experimental setup for mechanical oscillation linewidth measurements. Stokes and anti-Stokes fields are shown being coupled onto the tapered, optical fiber waveguide.

When no external gain is present and the system is solely thermally excited (i.e., $\delta \nu=\Delta \nu$ ), the energy of the resonator is determined by the equipartition theorem. This well-known principle in statistical mechanics states that in equilibrium with a thermal bath at temperature $T$, each degree of freedom (contributing a quadratic term in the Hamiltonian) contains an average energy of $(1 / 2) k_{B} T$. The total stored energy $(E)$ in a one-dimensional thermally driven harmonic oscillator (i.e., two degrees of freedom: kinetic energy $\propto \dot{x}^{2}$ and potential energy $\propto x^{2}$ ) is therefore $k_{B} T$. However, when $\delta \nu=\Delta \nu$, Eq. (4) predicts that the stored energy in the oscillator is $(1 / 2) k_{B} T$. This discrepancy can be explained by recalling the fact that although noise processes can perturb both the amplitude and phase of the oscillation, due to an inherent amplitude-limiting mechanism present in oscillator systems above threshold, amplitude fluctuations are suppressed and prevented from contributing to the linewidth. Therefore, only phase noise or one-half of the noise contribution remains in the system, explaining the origin of the factor of $1 / 2$ in Eq. (4). ${ }^{8}$

In a harmonic oscillator, where the energy of the oscillator is quadratically related to the amplitude of oscillations $\left[E=(1 / 2) m_{\mathrm{eff}} \Omega^{2} x^{2}\right]$, the linewidth of oscillation narrows in inverse quadratic relation to the amplitude of oscillation:

$$
\delta \nu=\Delta \nu\left(\frac{k_{B} T}{m_{\mathrm{eff}} \Omega^{2}}\right) \frac{1}{x^{2}},
$$

where $x$ is the amplitude of the mechanical oscillations. Both this behavior and the measurement of its magnitude (which allows one to infer a temperature) are used in this study to identify the underlying noise process.

The experimental setup consists of a microtoroid ${ }^{9}$ evanescently side coupled to a tapered optical fiber ${ }^{10}$ (see Fig. 1). A tunable narrow linewidth $(300 \mathrm{kHz})$ external cavity diode laser operating at telecommunication wavelengths $(1550 \mathrm{~nm})$ is used as the optical source. Desired coupling to one of the high quality optical modes of the microtoroid can be achieved by fine adjustments to the laser wavelength and polarization. Increasing the laser power to above the parametric threshold $\left(P_{\text {th }}\right)$ value initiates regenerative mechanical oscillations of the cavity structure, a signature of which is the modulated optical power transmitted beyond the waveguideresonator junction. ${ }^{1}$ This power modulation is detected using a photodiode (detection bandwidth of $125 \mathrm{MHz}$ ) and monitored with an oscilloscope. As described elsewhere, ${ }^{1-3}$ the optical modulation depth and consequently the photocurrent are directly related to the amplitude of vibrations (which causes variation in the optical path length for the circulating field within the cavity). An electrical spectrum analyzer is

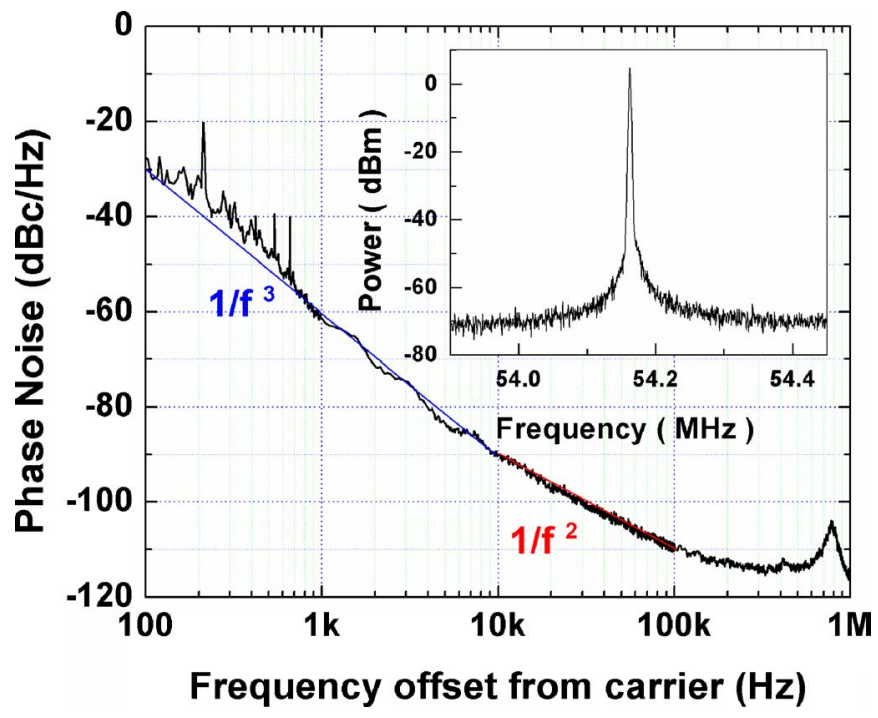

FIG. 2. (Color online) Typical, measured, phase noise, spectral density function of the optomechanical oscillator. Inset: An electrical power spectrum around the fourth eigenmechanical mode when the optical input power is about three times larger than $P_{\text {th }}$. Note that here the measured linewidth is limited by the resolution of the RF spectrum analyzer.

used to analyze the spectral power density of the photocurrent. The inset to Fig. 2 shows a typical power spectrum associated with a mechanical eigenmode oscillating near $54 \mathrm{MHz}$. A key point is that the shape of this electrical power spectrum carries the phase information of the optomechanical oscillation. This results because the small-signal photocurrent is produced by the mixing of the continuous-wave, optical pump with Stokes and anti-Stokes fields (see Fig. 1) and as such contains a replica of the mechanical motion (both amplitude and phase).

High-precision linewidth measurements could be performed using a phase noise analyzer system. As discussed earlier, for above threshold operation, amplitude fluctuations are damped and therefore phase noise plays the dominant role in the linewidth of an oscillator. The phase noise performance of an oscillator is best defined at different frequency offsets $(\Delta f)$ in terms of a phase noise spectral density function [defined as $\mathcal{L}(\Delta f)$ in the following discussion] measured relative to the oscillation frequency (the carrier frequency). It denotes the deviation of the oscillator performance from ideal behavior, and furthermore, the observed spectral dependences in this function can be used to ascertain information concerning the underlying nature of contributing noise mechanisms. ${ }^{11}$ Figure 2 shows a typical result of such a measurement on the optomechanical oscillator $\left(P>P_{\mathrm{th}}\right)$. It resembles the well-known behavior of a free-running oscillator. $^{12}$ Specifically, the $1 / f^{3}$ regime in Fig. 2 shows the cumulative effect of all the environmental noise processes that are also known as "flicker noise." These noise mechanisms are relatively slow and dominate the long-term performance of the device $(<\sim 10 \mathrm{kHz}$ frequency offsets). The $1 / f^{2}$ regime, on the other hand, is a signature of fast or almost instantaneous noise mechanisms and is associated with the Lorentzian profile line shape function. The relationship between the phase noise spectral density function of an oscillator in the $1 / f^{2}$ region and its Lorentzian linewidth $\delta \nu$ is given by Eq. (6), a discussion of which can be found in Ref. 12:

to AIP license or copyright, see http://apl.aip.org/apl/copyright.jsp 


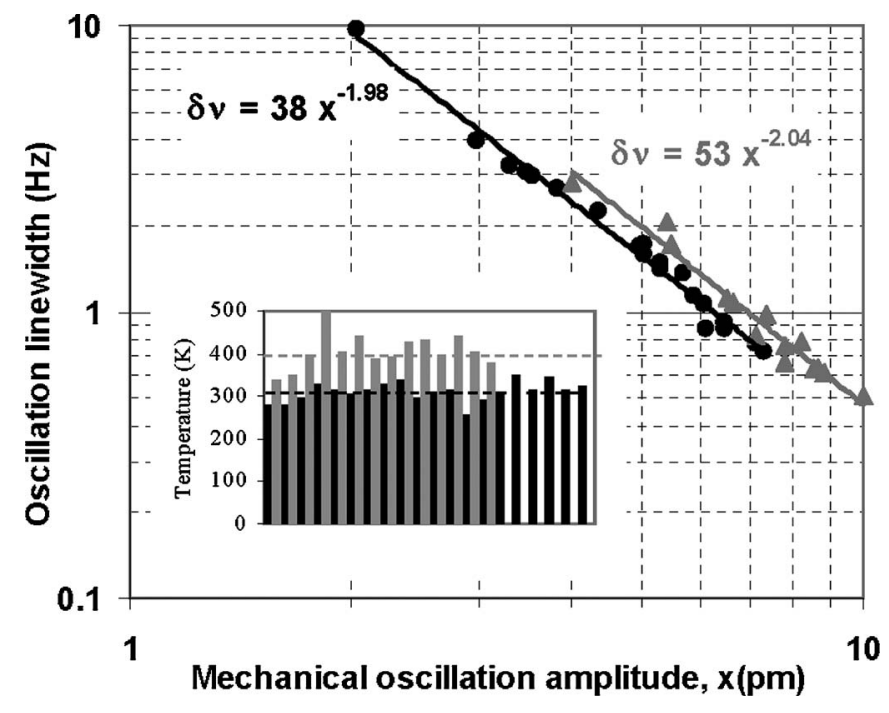

FIG. 3. Linewidth measurements of the optomechanical oscillator for different amplitudes of oscillation plotted in picometers. The measurement is done at room temperature (dots) and at $90{ }^{\circ} \mathrm{C}$ above room temperature (triangles). The solid lines and the corresponding equations are the best fits to the log-log data. The inset shows the derived temperatures [Eq. (5)]. The gray and black bars are extracted from high temperature data and room temperature data, respectively. (the dashed lines represent the average temperature for each case).

$$
\delta \nu=2 \pi \Delta f^{2} 10^{\mathcal{L} / 10},
$$

where $\Delta f$ is the frequency offset from the mechanical oscillation frequency (carrier frequency) and $\mathcal{L}$ has the units of decibels (referenced to the carrier) per hertz $(\mathrm{dBc} / \mathrm{Hz})$.

Using the above approach, we have measured oscillation linewidth as a function of mechanical oscillation amplitude. This was accomplished by fine adjusting the laser pump frequency in the proximity of the optical resonance, and thereby altering the amount of stored optical energy in the cavity as well as the magnitude of mechanical oscillations. Figure 3 gives the result of these measurements at two different temperatures. Evident from the data is the inverse quadratic dependence of the oscillation linewidth on the oscillation amplitude, in agreement with inverse-power linewidth narrowing [see Eq. (5)]. Using the measured parameters for the studied structure, one can extract effective temperatures at each data point in the graph using Eq. (5). The inset in Fig. 3 shows the temperatures derived from the data in the main figure. It is noteworthy that the average temperature from the first measurement (black dots and bars) is $312 \pm 20 \mathrm{~K}$, close to room temperature. This further confirms that the observed phase noise is thermomechanical in nature, i.e., Eq. (5).

To further verify the thermomechanical origin of the observed noise, the temperature of the microtoroid was increased by mounting the sample on a heating element. The linewidth measurement was repeated at $90^{\circ} \mathrm{C}$ above room temperature. The data for this measurement are also presented in Fig. 3 (gray triangles) and show that the linewidth versus amplitude graph is shifted upward in the log-log plot, demonstrating the higher level of noise in the system. The extracted temperatures from the high temperature graph are also shown in the inset (gray bars), and the average temperature is $400 \pm 25 \mathrm{~K}$, in agreement with the actual temperature of the structure $(390 \mathrm{~K})$. The above findings have been observed in other samples and verify the thermal equipartition origin of this noise.
In summary, this work has reported observation of the fundamental linewidth in a mechanical oscillator driven through radiation pressure. Our experimental results demonstrate that microtoroid optomechanical oscillators are currently operating at the Brownian-noise limit. Beyond the general significance of this measurement in the context of regenerative mechanical oscillators, the ability to observe and measure the thermal vibrations of a microstructure is beneficial for sensitive measurement of the amplitude fluctuations in a harmonic oscillator. Previously homodyne interferometric techniques were used to reveal minute vibrations of phase in a light wave used to probe the amplitude of a fluctuating mirror in a Fabry-Pérot cavity. ${ }^{13}$ In our measurement, however, the ability to characterize the device in the above threshold regime, where the structure is undergoing regenerative oscillations, has provided an alternate approach. The narrow bandwidth nature of the above threshold measurements filters out the environmental broadband noises and improves the signal to noise ratio. This approach can also reduce the complexity of the measurement by enabling the study of the noise processes through linewidth measurement in the rf domain.

This technique may also be used to study the quantum noise processes, such as back action noise, ${ }^{14}$ in optomechanical systems. ${ }^{5,14}$ The observation of these competing noise mechanisms becomes possible by reducing the thermal noise in the structure. Along these lines, we note that application of this measurement technique in a cryogenic vacuum environment has a twofold benefit regarding the reduction of the thermal noise: a reduction in the thermal bath temperature and the improvement of the mechanical quality factor. ${ }^{15}$

This work was supported by DARPA and the Caltech Lee Center.

${ }^{1}$ H. Rokhsari, T. J. Kippenberg, T. Carmon, and K. J. Vahala, Opt. Express 13, 5293 (2005)

${ }^{2}$ T. J. Kippenberg, H. Rokhsari, T. Carmon, and K. J. Vahala, Phys. Rev. Lett. 95, 033901 (2004).

${ }^{3}$ T. Carmon, H. Rokhsari, L. Yang, T. J. Kippenberg, and K. J. Vahala, Phys. Rev. Lett. 94, 223902 (2005).

${ }^{4}$ Here $r(t)$ represents the effective radial displacement of the microtoroid, $P_{\text {circ }}$ is the circulating optical power, $n$ is the effective refractive index of the optical mode, $c$ is the speed of light, $\gamma_{0}$ is the loss rate due to mechanical dissipation (assuming that the mechanical motion is subject to a frictional force with the frictional constant $\left.m_{\text {eff }} \gamma_{0}\right), m_{\text {eff }}$ is the effective resonator mass for radial motion, and $\Omega$ is the angular frequency of the mechanical oscillation. Note that $P_{\text {circ }}(r)$ is a function of the optical and mechanical quality factors as well as optical wavelength offset from resonance.

${ }^{5}$ K. Jacobs, I. Tittonen, H. M. Wiseman, and S. Schiller, Phys. Rev. A 60, 538 (1999).

${ }^{6}$ A. L. Schawlow and C. H. Townes, Phys. Rev. 112, 1940 (1958).

${ }^{7}$ A. Yariv and P. Yeh, Optical Electronics in Modern Communications (Oxford University Press, New York, 1997), p. 393.

${ }^{8}$ W. A. Edson, Proc. IRE 48, 1454 (1960).

${ }^{9}$ D. K. Armani, T. J. Kippenberg, S. M. Spillane, and K. J. Vahala, Nature (London) 421, 925 (2003).

${ }^{10}$ M. Cai, O. Painter, and K. J. Vahala, Phys. Rev. Lett. 85, 74 (2000).

${ }^{11}$ Y. Hadjar, P. F. Cohandon, C. G. Aminof, M. Pinard, and A. Heidman, Europhys. Lett. 47, 545 (1999).

${ }^{12}$ A. Hajimiri and T. H. Lee, The Design of Low Noise Oscillators (Kluwer Academic, Dordrecht, 1999), pp. 53 and 151.

${ }^{13}$ I. Tittonen, G. Breitenbach, T. Kalkbernner, T. Muller, R. Conradt, and S. Schiller, Phys. Rev. A 59, 1038 (1999).

${ }^{14}$ V. B. Braginsky and F. K. Khalili, Quantum Measurements, edited by K. S. Thorne (Cambridge University Press, Cambridge, 1992).

${ }^{15}$ F. R. Blom, S. Bouwstra, M. Elwenspoek, and J. H. J. Fluitman, J. Vac. Sci. Technol. B 10, 19 (1992). 